\title{
Clinical characteristics and progression of COVID-19 confirmed cases admitted to a single British clinical centre-a brief case series report
}

\author{
joanne conway $^{1}$, anna gould ${ }^{1}$, richard westley ${ }^{1}$, suneil raju ${ }^{1}$, anja oklopcic ${ }^{1}$, alex \\ broadbent $^{1}$, and Ahmed Hafiz ${ }^{1}$ \\ ${ }^{1}$ Affiliation not available
}

May 5, 2020

\begin{abstract}
Objectives: In December 2019, a pneumonia like illness was first reported in Wuhan-China caused by a new coronavirus named corona virus disease -2019 (COVID-19) which then spread to cause a global pandemic. Most of the available data in the literature is derived from Chinese cohorts and we aim to contribute the clinical experience of a single British clinical centre with the characteristics of a British cohort. Design: A prospective case series. Setting: A single clinical centre in the UK. Methods: We have collected the demographics and medical characteristics of all COVID-19 positive cases admitted over two-week period. All cases were diagnosed by PCR. Results: Total of 71 COVID-19 patients were included in this case series. Majority of patients $(75 \%)$ were [?]75 years old and $58 \%$ were men. Pre-existing comorbidities was common $(85 \%$ of patients). Most patients presented with respiratory symptoms such as fever (59\%), shortness of breath (56\%) and cough (55\%). Gastrointestinal symptoms were second most common presenting compliant such as diarrhoea (10\%) and abdominal pain (7\%). Opacification in chest X-rays were demonstrated in $45 \%$ of patients. All patients received supportive treatment and no specific antiviral therapy was administered in this cohort. So far, 18 (25\%) patients have fully recovered, 9 patients (13\%) escalated to a higher level of care and 10 (14\%) have died. Patients who died were non-significantly older than those who have recovered (78.0 v 69.2 years, $\mathrm{p}=0.15)$ but they had a significantly higher clinical frailty scores $(5.75 \mathrm{v} 3.36, \mathrm{p}=0.005)$. Conclusion: This case series demonstrated that the characteristics of British COVID-19 patients were generally similar to what is published in literature although we report more gastrointestinal symptoms at presentation. We have identified frailty as a risk factor for adverse outcome in COVID-19 patients and suggest that it should be included in the future vaccination recommendations.
\end{abstract}

\section{Introduction}

The respiratory disease COVID-19 was initially reported in Wuhan-Hubei province-China. ${ }^{1}$ The disease has since become a global pandemic. COVID-19 spreads rapidly by human-to-human transmission, primarily through respiratory droplets, physical contact and via fomites. ${ }^{2}$ Compared with influenza, COVID-19 is thought to have a higher reproductive number of 2-2.5 (more people will be infected per index case) but a longer serial interval of 5-6 days (compared with 3 days for influenza). ${ }^{3}$ COVID-19 typically presents with fever, myalgia, fatigue and dyspnoea or with less common gastrointestinal symptoms and headache. ${ }^{2}$ Chest imaging shows bilateral patchy shadows, and blood tests often reveal lymphopaenia. ${ }^{4}$ A fatality rate of $2.3 \%$ across all age groups, $14.8 \%$ in patients [?]80 years old and $8.0 \%$ in those $70-79$ of age has been reported. ${ }^{5}$ Mortality was found to be higher in patients with pre-existing comorbidities. ${ }^{5}$ Most of the available data in the literature is derived from Chinese cohorts of patients. The objective of this case series is to collect clinical data on patients' characteristics, clinical presentations, laboratory findings, and progression of patients admitted to a District General Hospital in the UK with confirmed COVID-19 tests. Our aim is to contribute the clinical experience of a single British clinical centre to the rapidly growing 
global data pool on this new life-threatening respiratory disease.

\section{Methods}

Design

A prospective case series.

Setting

District general hospital in UK. Patients are first admitted into acute medical unit (AMU) which accepts non-selective cases with acute medical conditions. The unit is open to other specialties input, if required, including access to intensive care unit (ICU) for severely ill patients. As a routine practice, a Do Not Attempt Resuscitation (DNAR) form is usually singed by a senior medical officer after discussion with the patients and their families.

Case definition

We have included all the COVID-19 confirmed cases admitted over 2 -week period from $23^{\text {rd }}$ March to $4^{\text {th }}$ April 2020. Initial screening for cases was undertaken in line with Public Health England (PHE) which identifies suspected case as those presenting with clinical or radiological evidence of pneumonia, fever and respiratory symptoms. ${ }^{6}$

Case confirmation

Patients meeting the above criteria then had nasopharyngeal and oropharyngeal swabs for identification of 2019-nCoV nucleic acid by reverse transcription polymerase chain reaction (RT-PCR). Patients who tested negative by RT-PCR but still have a high index of clinical suspicion were re-tested with a further swab for RT-PCR. All suspected patients had an initial chest X-ray, some had a CT scan based on clinical requirement. CT scan was not used as a diagnostic tool and all of the patients had a RT-PCR confirmed diagnosis.

Data collection

We identified all COVID-19 cases from our laboratory data-base which has been followed up on a daily basis. Medical records were reviewed and patients' demographic, medical and social history was collected. Laboratory and radiological information were available in the electronic records. We used a standardised data collection sheet which was used by all the authors. To assess frailty, we used the clinical frailty score (CFS) which classify patients in a range of very fit (score 1) to terminally ill (score 9). ${ }^{7}$

\section{Statistical analysis}

Continuous variables are presented as means and standard deviations (SD) and categorical ones as percentages. The t-test $t$ test was used for continuous variables and two-sided $\mathrm{p}$ value of $<0.05$ was considered significant. We displayed descriptive statistics using the statistical software package StataCorp. 2019. Stata Statistical Software: Release 16. College Station, TX: StataCorp LLC.

\section{Results}

Total of 71 COVID-19 confirmed consecutive cases who were admitted to hospital over 2 week period were included. Patient demographics are displayed in Table 1. Majority (75\%) of patients were [?]65 years old, $58 \%$ were men and the source of infection was unidentified in the majority of cases (77\%). Likely the transmission was human to human as no animal contact was recorded. Five patients reported that they had contact with a symptomatic family member, 3 returned to the UK from a holiday ( 2 from Spain and 1 from a Mediterranean cruise). Eight patients were admitted for other medical conditions and developed symptoms and tested positive while in-hospital. Most of the patients (85\%) had pre-existing comorbidities, with hypertension (45\%) most common, followed by cardiovascular disease (34\%), chronic obstructive pulmonary disease $(33 \%)$ and diabetes (23\%). Presenting symptoms and clinical observations on the day of admission are displayed in Table 2. Respiratory symptoms were the most common presenting complaint such as fever $(59 \%$ of patients), shortness of breath $(56 \%)$ and cough $(55 \%)$. Gastrointestinal symptoms were the second most 
common such as diarrhoea (10\%) and abdominal pain (7\%). Most patients (66\%) were hypoxic and required oxygen therapy on admission. Initial laboratory results and imaging are displayed in Table 3. Leucocytosis associated with lymphopenia was the most common laboratory abnormality. Opacifications in chest X-rays were demonstrated in 32 patients. Seven patients had a CT-chest due to worsening of their condition. All of the CT scans demonstrated ground glass changes. All patients received supportive treatment but no specific antiviral therapy was administered. Available outcome data is displayed in Table 4. Patients who died ranged from 66-90 years old and were none significantly older than those who have recovered and been discharged (average age 78.0 v 69.2 years, $\mathrm{p}=0.15$ ) but they had a significantly higher clinical frailty scores $(5.75$ $3.36, \mathrm{p}=0.005)$.

\section{Discussion}

We present the first UK clinical data of 71 cases of COVID-19 patients admitted to one clinical centre. The majority of patients (70 patients) were white British and one patient was Asian. Our cohort of patients of mean (SD) age of 70.7 (16.6) years, with $75 \%$ are [?]65 years, appears older than the published Chinese cohort \{median age was only 46.0 years and the proportion of older patients ( $>60$ years) was $44.1 \%$, we had slightly more men $(58 \%$ v $51.6 \%)$ but the comorbidities were similar. ${ }^{8}$ Around $10 \%$ of our patients presented with gastrointestinal symptoms such as diarrhoea compared to only $3.8 \%$ in a Chinese study. ${ }^{9}$ Although in another Chinese study by Wang et al, $10 \%$ of patients initially presented with nausea and diarrhoea 1-2 days before the onset of fever and dyspnoea. ${ }^{4}$ Laboratory systemic abnormalities particularly lymphopenia and elevated CRP and chest imaging findings are consistent with previously published literature. ${ }^{10}$ The majority of our patients $(55 \%)$ had normal chest x-ray in comparison to only around $18 \%$ in a Chinese report. ${ }^{9}$ Our case fatality rate of $14 \%$ was similar to $8 \%$ for those aged $70-79$ and $14.8 \%$ for those [?] 80 years that was reported from China. ${ }^{8}$ Also, previously, older age has been reported as an important independent predictor of mortality in SARS and MERS. ${ }^{11,12}$ Older people are more susceptible to infection likely due to associated comorbidities and attenuation of the innate immunity. ${ }^{13}$ The ageing immune system is characterized by a low grade and chronic systemic inflammatory state or "InflammAgeing" and is associated with an increased susceptibility to infection. ${ }^{14}$ One important finding in this case series, which has not been reported yet in the literature, is the emergence of frailty as a detrimental significant factor for poor outcome in patients with COVID-19. Frailty is a syndrome that is characterised by multisystem dysregulation that leads to reduced physiologic reserve and increased risk of adverse health outcomes. Dysregulation in the innate and adaptive immunity also leads to chronic inflammation, with increase in inflammatory markers, and increased susceptibility to severe infections. Increased inflammatory markers have been shown in patients with viral pneumonia. ${ }^{13}$ Although frailty was not formally assessed in the COVID-19 studies, the fact that old age is associated with comorbidities and was associated with an increased risk of COVID-19 infection and worse outcomes may suggest underlying frailty was common in these old patients. The strength of this case series is its prospective design which allowed follow up of cases as well as the use of electronic record which allowed accurate documentation of results. However, this study has few limitations which include first, the small number included so far and the incomplete conclusion of outcomes of the patients who are still under treatment in hospital however, this series is in a continuous follow up and successive publications will follow as more information are gathered. Second, we were not able to record the incubation period for the current infection as this was not clearly re-called by the patients. Third, we are not able to report efficacy of any specific antiviral therapy as per our hospital protocol, this has not been used.

\section{Conclusion}

This case series demonstrated that the characteristics of British COVID-19 patients were generally similar to what is published so far in literature although we report more gastrointestinal symptoms at presentation and more normal chest X-ray findings in our cohort. We have also identified frailty as an emergent risk factor for adverse outcome in COVID-19 patients and suggest that it should be included in the future vaccination recommendations.

\section{References}


1) Li Q, Guan X, Wu P, et al. Early Transmission Dynamics in Wuhan, China, of Novel Coronavirus-Infected Pneumonia. NEJM 2020; 382: 1199-207.

2. COVID-19: epidemiology, virology and clinical features. GOV.UK. 2020 [Date of Access: 8 April 2020]. URL: https://www.gov.uk/government/publications/wuhan-novel-coronavirus-backgroundinformation/wuhan-novel-coronavirus-epidemiology-virology-and-clinical-features.

3. Q\&A: Similarities and differences - COVID-19 and influenza. World Health Organization 2020 [Date of Access: 8 April 2020]. URL: https://www.who.int/news-room/q-a-detail/q-a-similarities-and-differencescovid-19-and-influenza

4. Wang $\mathrm{D}, \mathrm{Hu} \mathrm{B}, \mathrm{Hu} \mathrm{C}$, et al. Clinical Characteristics of 138 Hospitalized Patients With 2019 Novel Coronavirus-Infected Pneumonia in Wuhan, China. JAMA 2020; 323: $1061 . \quad$ URL: https://www.ncbi.nlm.nih.gov/pubmed/32031570.

5. Wu Z, McGoogan J. Characteristics of and Important Lessons From the Coronavirus Disease 2019 (COVID-19) Outbreak in China. JAMA 2020; 323: 1239. Available from: https://jamanetwork.com/journals/jama/fullarticle/2762130.

6. COVID-19: investigation and initial clinical management of possible cases [Internet]. GOV.UK. 2020 [cited 8 April 2020]. Available from: https://www.gov.uk/government/publications/wuhan-novelcoronavirus-initial-investigation-of-possible-cases/investigation-and-initial-clinical-management-of-possiblecases-of-wuhan-novel-coronavirus-wn-cov-infection

7. K. Rockwood et al. A global clinical measure of fitness and frailty in elderly people. CMAJ 2005; 173 : 489-95.

8. The novel coronavirus pneumonia emergency response epidemiology team. The epidemiological characteristics of an outbreak of 2019 novel coronavirus diseases (COVID-19) - China, 2020. China CDC Weekly. 2020; 2: 113-22.

9. Guan W, Ni Z, HU Y, et al. Clinical Characteristics of Coronavirus Disease 2019 in China. NEJM 2020; DOI: $10.1056 /$ NEJMoa2002032.

10. Huang C, Wang Y, Li X, et al. Clinical features of patients infected with 2019 novel coronavirus in Wuhan, China. Lancet 2020; 395: 497-506.

11. Choi KW, Chau TN, Tsang O, et al. Outcomes and prognostic factors in 267 patients with severe acute respiratory syndrome in Hong Kong. Ann Intern Med 2003; 139: 715-23.

12. Hong K-H, Choi J-P, Hong S-H, et al. Predictors of mortality in Middle East respiratory syndrome (MERS). Thorax 2018; 73: 286-9.

13. Guo L, Wei D, Zhang X, et al. Clinical Features Predicting Mortality Risk in Patients With Viral Pneumonia: The MuLBSTA Score. Front Microbiol 2019; 10: 2752.

14. Franceschi C, Bonafe M, Valensin S, et al. Inflamm-aging. An evolutionary perspective on immunosenescence. Ann N Y Acad Sci 2000; 908: 244-54.

\begin{tabular}{lllr}
\hline Table 1: Patients Demographics. & Table 1: Patients Demographics. & Table 1: Patients Demographics. & N \\
\hline Personal Demographics & & Total number & 71 \\
& Age (years) & Mean (SD) & 70 \\
& & $18-64$ & 53 \\
& & $65+$ & 24 \\
& Sex & Range & 41 \\
& Race & Male & 70
\end{tabular}




\begin{tabular}{llll}
\hline Table 1: Patients Demographics. & Table 1: Patients Demographics. & Table 1: Patients Demographics. & Nu \\
\hline & \multirow{2}{*}{ CFS } & Asian & 1 \\
& & Mean (SD) & 4.1 \\
& \multirow{2}{*}{ Source } & $<5$ & 42 \\
& & $5+$ & 29 \\
& & Unknown & 85 \\
& \multirow{2}{*}{ Pre-existing conditions } & Hospital acquired & 5 \\
& & Family member & 3 \\
& & Recent travel & 60 \\
& & Any & 32 \\
& HTN & 24 \\
& & CVD & 23 \\
& COPD & 16 \\
& DM & 10 \\
& Smoking status & Dementia & 9 \\
& & Renal disease & 5 \\
& Liver disease & 38 \\
& Unknown & 20 \\
& Ex-smoker & 13 \\
& Never smoked & 0 \\
\hline
\end{tabular}

CFS=Clinical frailty score, HTN=Hypertension, $\mathrm{DM}=$ Diabetes mellitus, $\mathrm{COPD}=$ Chronic obstructive pulmonary disease, $\mathrm{CVD}=$ Cardiovascular disease.

Table 2: Presenting symptoms and initial observations.

Clinical Presentation
Table 2: Presenting symptoms and initial observations.

Symptoms

Table 2: Presenting symptoms and initial observations.

Fever

SOB

Cough

Fatigue

Phlegm

Vomiting

New confusion

Diarrhoea

Generally unwell

Myalgia

Abdominal pain

Headache

Chest pain

Sore Throat

Fall

Collapse

Anosmia

Poor oral intake

Haemoptysis

Change in taste
Number of Patients (\%)

$42(59)$

$40(56)$

$39(55)$

$16(23)$

$9(12.6)$

8 (11)

$8(11)$

$7(9.8)$

$7(9.8)$

$6(8.5)$

$5(7)$

$4(5.6)$

4 (5.6)

2 (2.8)

2 (2.8)

$2(2.8)$

2 (2.8)

1 (1.4)

1 (1.4)

1 (1.4) 


\begin{tabular}{llll}
\hline $\begin{array}{l}\text { Table 2: Presenting } \\
\text { symptoms and } \\
\text { initial observations. }\end{array}$ & $\begin{array}{l}\text { Table 2: Presenting } \\
\text { symptoms and } \\
\text { initial observations. }\end{array}$ & $\begin{array}{l}\text { Table 2: Presenting } \\
\text { symptoms and } \\
\text { initial observations. }\end{array}$ & $\begin{array}{l}\text { Number of Patients } \\
(\%)\end{array}$ \\
\hline Initial Observations & Temperature (Celsius) & $<37.5$ & $29(41)$ \\
& & $37.5+$ & $42(59)$ \\
& Oxygen Sats & $<96 \%$ & $47(66)$ \\
& RR & $96 \%+$ & $24(34)$ \\
& \multirow{2}{*}{ Systolic BP (mmHg) } & $<20$ & $18(25)$ \\
& \multirow{2}{*}{ HR } & $20+$ & $53(75)$ \\
& & $120+$ & $21(29.5)$ \\
& $<100$ & $50(70.5)$ \\
& $100+$ & $48(67.7)$ \\
\hline
\end{tabular}

$\mathrm{SOB}=$ Shortness of breath, $\mathrm{RR}=$ Respiratory rate, Sats=Saturation, $\mathrm{BP}=\mathrm{Blood}$ pressure, $\mathrm{HR}=$ Heart rate.

\begin{tabular}{lll}
\hline Table 3: Lab results and imaging. & Table 3: Lab results and imaging. & Table 3: Lab results and imaging. \\
\hline Investigations & Blood results & Total WCC \\
& Neutrophil \\
& Lymphocyte \\
& Eosinophil \\
& Hb Creatinine \\
& Urea $^{+}$ \\
& $\mathrm{Na}^{+}$ \\
& $\mathrm{ALT}$ \\
& $\mathrm{AST}$ \\
& $\mathrm{CRP}$ \\
& \\
& & Clear \\
& Unilateral consolidation \\
& $\mathrm{B} / \mathrm{L}$ consolidation \\
\hline
\end{tabular}

$\mathrm{WCC}=$ White cell count, $\mathrm{Hb}=$ Haemoglobin, $\mathrm{ALT}=$ Alanine aminotransferase, AST $=$ Aspartate aminotransferase, $\mathrm{CRP}=\mathrm{C}$-reactive protein, $\mathrm{XR}=\mathrm{X}$-Ray, $\mathrm{CT}=$ Computerised tomography, $\mathrm{B} / \mathrm{L}=$ Bilateral.

\begin{tabular}{lllll}
\hline & & Outcomes & Outcomes & Outcomes \\
\hline Table 4: Patients outcomes & Table 4: Patients outcomes & Home & HDU/ICU & Death \\
& Number (\%) & $18(25)$ & $9(13)$ & $10(14)$ \\
Average & Age (years) & 69.2 & 61.2 & 78 \\
& Clinical frailty score* & 3.36 & 2.56 & 5.75 \\
& Total WCC & 8.89 & 9.28 & 5.46 \\
& Neutrophil & 7.26 & 8.1 & 4.33 \\
& Lymphocytes & 0.87 & 0.73 & 0.58 \\
& & Outcomes (\%) & Outcomes (\%) & Outcomes (\%)
\end{tabular}




\begin{tabular}{lllll}
\hline & & Outcomes & Outcomes & Outcomes \\
\hline \multirow{3}{*}{ Number of patients } & & Home & HDU/ITU & Death \\
& No co-morbidities & $4(22)$ & $4(44)$ & 0 \\
& Any co-morbidities & $14(78)$ & $5(56)$ & $10(100)$ \\
& Any XR findings & $15(21)$ & $9(100)$ & $6(60)$ \\
& Normal XR & $3(17)$ & 0 & $4(40)$ \\
& Needed oxygen & $11(61)$ & $7(78)$ & $6(60)$ \\
\hline
\end{tabular}

HDU/ICU =High-dependency unit/intensive care unit, ${ }^{*}$ significant. 